\title{
Limb Versus Precordial ECG Leads as Improved Predictors of Electrical Cardioversion Outcome in Persistent Atrial Fibrillation
}

\author{
Eva M Cirugeda ${ }^{1}$, Sofía Calero ${ }^{2}$, Aurelio Quesada $^{3}$, Víctor M Hidalgo ${ }^{2}$, José J Rieta ${ }^{4}$, Raúl Alcaraz $^{1}$ \\ ${ }^{1}$ Research Group in Electronic, Biomedical and Telecommunications Engineering, \\ University of Castilla La-Mancha, Cuenca, Spain \\ ${ }^{2}$ Cardiac Arrhythmia Department, University Hospital of Albacete, Spain \\ ${ }^{3}$ Cardiology Department, Hospital General Universitario, Valencia, Spain \\ ${ }^{4}$ BioMIT.org, Electronic Engineering Department, Universtat Politécnica de Valencia, Spain
}

\begin{abstract}
Electrical cardioversion (ECV) is an effective and lowcost rhythm control strategy for persistent atrial fibrillation (AF). Because of its limited mid- and long-term success rates, prediction of early failure could avoid patients with reduced chance to maintain sinus rhythm (SR). To this end and due to its proximity to the right atrium, several indices characterizing atrial activity have been proposed based on lead V1. However, information from other leads has been discarded to date. Hence, this work studies how effective some common indices computed over the whole set of 12 standard ECG leads are in predicting ECV outcome. Precisely, amplitude, dominant frequency, and sample entropy were computed from the fibrillatory $(f-)$ waves extracted for each one of 12 standard leads acquired before $E C V$ from 58 patients in persistent $A F$. The classification between the patients who relapsed to $A F$ and maintained sinus rhythm after a follow-up of 4 weeks achieved by these parameters was better from limb lead II than from $V 1$, thus reporting improvements about 6 and $12 \%$. As a consequence, characterization of $f$-waves from the more accessible limb lead II has proven to be the best choice to improve AF ECV outcome prediction from the ECG.
\end{abstract}

\section{Introduction}

Atrial fibrillation (AF) is a supra-ventricular tachyarrhythmia with extremely rapid and uncoordinated atrial activations, often associated with structural heart diseases and other co-occurring chronic conditions, including obesity, apnea, and hypertension [1]. Even though AF is not life-threatening in itself, it can cause haemodynamic abnormalities leading to thromboembolism and stoke [2]. Indeed, patients suffering from AF present a 5-fold risk of stroke and a 2-fold risk of death and dementia compared to healthy people of the same age $[1,3]$.
When AF lasts for more than seven days, it is termed as persistent $\mathrm{AF}$ and often requires an external intervention for its termination. Although sinus rhythm (SR) restoration is associated with more hospitalizations than maintaining AF with a controlled heart rate, it reaches improvements in symptoms and quality of live, and it is therefore pursued in most patients [1]. For that purpose, current guidelines about AF management recommend electrical cardioversion (ECV) as one possible strategy for many subjects [1]. This is a low-cost and high effective procedure, which is able to initially restore SR in almost $90 \%$ of the patients [4]. However, mid- and long-term rates of AF recurrence after ECV are still large, since the arrhythmia recurs in about $20-40 \%$ of the patients within the first month, and in about $60-80 \%$ during the first year [4]. Additionally, the procedure does not involve major complications, but it can be responsible for some annoying sideeffects, such as post-shock bradycardia, malignant ventricular arrhythmias, sedation-related hassles, arterial thromboembolism, or hypotension [1]. Hence, anticipation of ECV outcome is an interesting clinical challenge, because tailored decisions about whether this treatment is the most adequate choice for each patient could be enabled [5].

So far, some indices have been proposed as predictors of ECV outcome [6,7]. Most of them are based on characterizing the fibrillatory $(f-)$ waves reflected on the surface ECG. For instance, indices such as $f$-waves amplitude (FWA), their regularity (estimated via Sample Entropy) or their dominant frequency (DF) have reported promising predictions of ECV outcome, when they were computed from standard lead V1 $[6,7]$. However, considering only this lead to anticipate the procedure result discards other spatiotemporal information about the cardiac dynamics supporting the arrhythmia. Hence, this work studies how effective the aforementioned indices computed over the whole set of 12 standard ECG leads are in predicting ECV outcome with respect to just V1. 
Table 1. Clinical characteristics for the patients considered in the study. Information is separtelly presented for the patients who maintained SR and relapsed to AF.

\begin{tabular}{lcc}
\hline \hline Parameter & SR maintenance & AF relapse \\
\hline Patients & 27 & 31 \\
Men / Women & $15 / 12$ & $18 / 12$ \\
Underlying heart disease & 9 & 10 \\
Left atrial diameter $(\mathrm{mm})$ & $47.32 \pm 4.76$ & $44.72 \pm 7.32$ \\
\hline \hline
\end{tabular}

\section{Methods}

\subsection{Study population}

A total of 58 patients diagnosed with persistent AF, under antiarrhythmic drug treatment, and indicated for ECV were considered in the study. Before and during the whole ECV procedure, a 12-lead ECG signal was continuously recorded at a sampling rate of $1024 \mathrm{~Hz}$ and 16 bit resolution. The ECV protocol consisted of the application of a maximum of four synchronized electrical shocks over the patient's thorax following an increasing sequence of 200, 300,360 and $360 \mathrm{~J}$, respectively. All patients reverted to SR during the procedure, but 31 of them relapsed to AF during the first 4 weeks. Most relevant clinical information for these subjects can be found in Table 1.

\subsection{Preprocessing of the ECG signal}

Leads in the ECG signal were separately preprocessed to reduce baseline wander, powerline interference and high frequency noise. Similarly, R-peaks were independently detected for each lead making use of a previous algorithm [8]. Nonetheless, mistakes in this procedure were automatically identified by comparing timings for R-peaks detected in each lead and then manually corrected.

Next, the $f$-waves found in each lead were extracted using a previously published QRST cancellation method [9]. In short, QRS complexes were aligned to their R-peak and clustered based on a beat morphology template matching algorithm. A beat was considered to belong to a class when the cross-correlation coefficient was above 0.8 [6]. QRST cancellation was then performed in a recursive way, starting from the smaller cluster and following an increasing order. The QRST segment duration was set to the minimum value between $470 \mathrm{~ms}$ (typical value) and $90 \%$ of the median RR interval. Finally, the resulting signal contained the $f$-waves and was high-pass filtered at $3 \mathrm{~Hz}$ for removing all ventricular residua.

\subsection{Atrial activity characterization}

Three indices widely used to characterize $f$-waves, including FWA, DF and sample entropy (SampEn) [6, 7], were separately computed for each one of 12 leads. Thus, considering $f(n)$ as $N$ sample-length signal containing the $f$-waves and $n=1: N$, FWA was estimated as [7]:

$$
F W A=\sqrt{\frac{1}{N} \sum_{n=1}^{N}|f(n)|^{2}}
$$

The DF was obtained from the averaged power spectral density (PSD) for the $f$-waves as the frequency with the highest amplitude within the frequency interval of $3-$ $12 \mathrm{~Hz}[6]$, so that

$$
D F=\arg \left\{\max _{f_{k}=3-12 H z}\left\{\overline{P S D}\left(f_{k}\right)\right\}\right\} .
$$

The averaged PSD was estimated as the mean of the individual PSD whose cross correlation coefficient was above 0.7. Individual PSD was obtained from successive 6 second-length segments of the $f$-waves using a Welch Periodogram. Note that an overlapping of 4 seconds was considered between successive segments.

Finally, the $f$-waves regularity was assessed by computing SampEn over their main component, $f f(n)$. This signal was obtained by filtering $f(n)$ with a band-pass structure with a 5-Hz bandwidth centered on the DF [7]. SampEn was originally defined to deal with physiological signals [10], and it belongs to a family of statistics designed to account for the regularity inherent to a nonlinear timeseries. This entropy is defined as the logarithmic likelihood ratio that two sequences of length $m$ that are similar within a distance $r$ will remain similar for an incremental sequence length of one unit [10]. Its computation follows the next steps [10], i.e.:

1. Obtain the epochs $v_{m}(n)$ of length $m$, which are defined as

$$
v_{m}(n)=\{f f(n+i): 0 \leq i \leq m-1\} .
$$

2. Estimate the number of similar epochs of length $m$ within a distance $r$ following the Chebyshev distance, i.e.

$$
B_{k}^{m}(r)=\frac{1}{N-m-1} \sum_{\substack{j=1 \\ j \neq k}}^{N-m}\left(d_{j k}(m)<r\right),
$$

where

$$
d_{j k}(m)=\max \left\{\left|v_{m}(j)-v_{m}(k)\right|\right\} .
$$

3. Compute the probability that two epochs of length $m$ will match, so that

$$
B^{m}(r)=\frac{1}{N-m} \sum_{k=1}^{N-m} B_{k}^{m}(r),
$$


4. Increase the sequence length in one unit, $m+1$, and repeat steps (1)-(3) to estimate SampEn as

$$
\operatorname{SampEn}(f f, m, r, N)=-\ln \frac{B^{m+1}(r)}{B^{m}(r)} .
$$

It should be noted that SampEn was here computed on non-overlapping segments of 30 second-length. Moreover, the parameters $m$ and $r$ were set to 2 and 0.2 times the standard deviation of $f f(n)$, respectively, such as recommended in previous works [10].

\subsection{Statistical Analysis}

The statistical separability between the patients who relapsed to AF and maintained SR was assessed by a Student's $t$-test or a Wilconxon rank sum test, depending on whether data were normally distributed or not. Data normality was determined by a Kolmogorov-Smirnov test. On the other hand, the predictive capability of each analyzed parameter was evaluated by means of a receiver operating characteristic (ROC) curve [11]. This plot provides information on the sensitivity (Se) and specificity ( $\mathrm{Sp}$ ) of an index when used as a classifier. Se indicates the ratio of patients relapsing to AF correctly identified, while Sp determines the percentage of patients maintaining SR correctly identified. These two values were determined according to the Youden's criteria, and the area under the ROC curve (AROC) was also computed as an aggregate measure of performance across all possible classification thresholds.

\section{Results}

Fig. 1 presents the boxplot distribution of FWA, DF and SampEn computed over the lead exhibiting the largest AROC. As can be seen, FWA provided higher values and a wider interquartile range for the patients maintaining SR than for those relapsing to AF. Contrarily, DF and SampEn obtained higher values for the patients who relapsed to AF, but a wider interquartile range was still noticed for those maintaining SR.

On the other hand, Table 2 displays values of AROC and statistical significance ( $p$-value) for the three indices separately computed from the 12 leads. Bold letters denote the largest AROC for each parameter. It can be noticed that the best performance for the three indices was found over the limb leads. More precisely, the highest discriminant ability for DF was reached on lead aVL, with an AROC of $81.1 \%$ ( $\mathrm{Se}=83.9 \%$ and $\mathrm{Sp}=74.1 \%$ ). This result outperformed the one obtained from lead V1 by around 6\% $(\mathrm{AROC}=75.7 \%, \mathrm{Se}=80.6 \%$ and $\mathrm{Sp}=63.0 \%)$. Regarding SampEn, the best classification result was obtained from lead II with an AROC of $78.7 \%$ ( $\mathrm{Se}=67.7 \%$ and $\mathrm{Sp}=$ $81.5 \%$ ), thus improving by $4 \%$ the one provided from lead
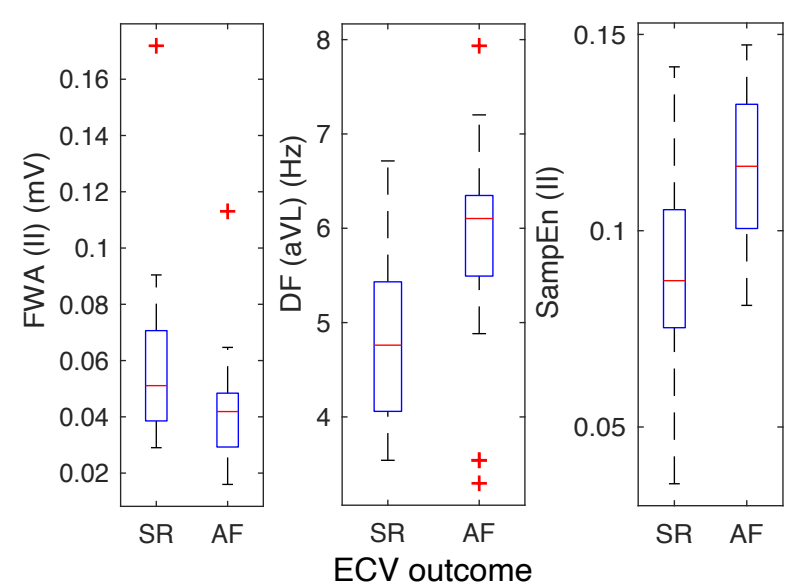

Figure 1. Boxplot distribution of (a) FWA on lead II, (b) DF over lead aVL, (c) SampEn on lead II.

V1 $($ AROC $=74.7 \%, \mathrm{Se}=80.6 \%$ and $\mathrm{Sp}=63.0 \%) . \mathrm{Sim}-$ ilarly, the best performance of FWA was noticed for lead II, with an AROC of $69.5 \%(\mathrm{Se}=70.4 \%$ and $\mathrm{Sp}=58.1 \%)$, thus outperforming more than $11 \%$ the one reported from lead V1 $($ AROC $=57.6 \%, \mathrm{Se}=40.7 \%$ and $\mathrm{Sp}=82.1 \%)$. Finally, note that FWA only provided statistically significant differences between groups of patients for leads II and III, while DF and SampEn did it for almost all leads.

\section{Discussion and conclusions}

In most of the previous works dealing with prediction of ECV outcome [12,13], analysis of lead V1 has been generally preferred due to its proximity to the right atrium [12]. However, the results obtained in the present work have shown that common parameters FWA, DF and SampEn achieved a better performance from limb leads, and particularly from lead II. This finding is not completely surprising due to two reasons. On the one hand, lead II can capture information from both right and left atria, because it is aligned with the interatrial septum [14]. In this way, $f$-waves in this lead could reflect more globally atrial activity and its organization. On the other hand, in contrast to unipolar leads (such as V1), lead II is a bipolar recording, thus providing a better signal-to noise ratio. Moreover, this signal has also been suggested to display the largest Pwaves [6]. Then, considering that $\mathrm{P}$-waves are replaced by $f$-waves during AF, it could be suggested that lead II also exhibits large fibrillatory activity.

It is also interesting to note that some recent studies have also analyzed the ability of FWA, DF and SampEn to anticipate the result of pharmacological cardioversion [15] and catheter ablation [16] from all standard leads. Despite the notable differences between these studies, they have also suggested that limb leads offer better results than V1. 
Table 2. Values of AROC and statistical significance ( $p$-value) obtained for the analyzed indices FWA, DF and SampEn when computed from the 12 standard ECG leads.

\begin{tabular}{llcccccccccccc}
\hline \hline & Parameter & I & II & III & aVR & aVL & aVF & V1 & V2 & V3 & V4 & V5 & V6 \\
\hline \multirow{2}{*}{ AROC } & FWA & 0.597 & $\mathbf{0 . 6 9 5}$ & 0.656 & 0.621 & 0.680 & 0.658 & 0.576 & 0.559 & 0.542 & 0.562 & 0.618 & 0.645 \\
& DF & 0.711 & 0.783 & 0.777 & 0.698 & $\mathbf{0 . 8 1 1}$ & 0.802 & 0.757 & 0.724 & 0.716 & 0.702 & 0.667 & 0.644 \\
& SampEn & 0.646 & $\mathbf{0 . 7 8 7}$ & 0.663 & 0.648 & 0.731 & 0.762 & 0.747 & 0.665 & 0.650 & 0.667 & 0.694 & 0.669 \\
\hline \multirow{2}{*}{$p$-value } & FWA & 0.207 & $\mathbf{0 . 0 1 1}$ & 0.043 & 0.115 & 0.019 & 0.040 & 0.326 & 0.445 & 0.585 & 0.427 & 0.127 & 0.059 \\
& DF & 0.006 & 0.001 & 0.001 & 0.010 & $\mathbf{0 . 0 0 1}$ & 0.001 & 0.001 & 0.004 & 0.005 & 0.009 & 0.030 & 0.061 \\
& SampEn & 0.057 & $\mathbf{0 . 0 0 1}$ & 0.034 & 0.055 & 0.003 & 0.001 & 0.001 & 0.031 & 0.051 & 0.030 & 0.012 & 0.028 \\
\hline \hline
\end{tabular}

On the other hand, it should also be remarked that the values of FWA, DF and SampEn obtained from lead II maintained the same trends as those obtained from lead V1 [7, 13]. In fact, in the present work higher values of FWA and lower values of DF and SampEn has still been noticed from lead II for the patients who maintained SR than for those who relapsed to AF. Hence, these results also agree the previous supposition that the presence of disorganized and low amplitude $f$-waves could be indicative of early failure of ECV [7, 13].

To sum up, characterization of $f$-waves from the more accessible limb leads than V1, particularly from II and aVL, seems to be a better choice to improve AF ECV outcome prediction from the surface ECG.

\section{Acknowledgements}

This research was funded by the projects DPI2017-83952C3 from MINECO/AEI/FEDER EU, SBPLY/17/180501/ 000411 from "Junta de Castilla La Mancha" and AICO/2019/036 from "Generalitat Valenciana".

\section{References}

[1] January C, et al. 2014 AHA/ACC/HRS guideline for the management of patients with atrial fibrillation: A report of the american college of cardiology/american heart association task force on practice guidelines and the heart rhythm society. Circ 2014;130(23).

[2] Duarte R, et al. Thrombin generation and other hemostatic parameters in patients with atrial fibrillation in use of warfarin or rivaroxaban. J Thromb Thrombolysis 2020;.

[3] Khoo C, et al. Atrial fibrillation, arrhythmia burden and thrombogenesis. Int J Cardiol 2012;157(3):318-23.

[4] Duytschaever M, Haerynck F, Tavernier R, Jordaens L. Factors influencing long term persistence of sinus rhythm after a first electrical cardioversion for atrial fibrillation. Pacing Clin Electrophysiol Jan 1998;21(1 Pt 2):284-7.

[5] Fujimoto Y, Yodogawa K, Maru YJ, Oka E, et. al. Advanced interatrial block is an electrocardiographic marker for recurrence of atrial fibrillation after electrical cardioversion. Int J Cardiol Dec 2018;272:113-117.

[6] Corino V, et al. Signal processing methods for informa- tion enhancement in atrial fibrillation: Spectral analysis and non-linear parameters. Biomed Signal Process Control 2006;1(4):271-281.

[7] Alcaraz R, et al. Noninvasive time and frequency predictor of long-standing atrial fibrillation early recurrence after electrical cardioversion: predictor of cardioversion outcome. Pacing Clin Electrophysiol 2011;34(10):1241-1250.

[8] Vest AN, Da Poian G, Li Q, Liu C, Nemati S, Shah AJ, Clifford GD. An open source benchmarked toolbox for cardiovascular waveform and interval analysis. Physiol Meas 10 2018;39(10):105004.

[9] Alcaraz R, et al. Adaptive singular value cancelation of ventricular activity in single-lead atrial fibrillation electrocardiograms. Physiol Meas 2008;29(12):1351-69.

[10] Richman J, Moorman J. Physiological time-series analysis using approximate entropy and sample entropy. Am J Physiol Heart Circ Physiol 2000;278(6):H2039-H2049.

[11] Zweig M, et al. Receiver-operating characteristic (ROC) plots: a fundamental evaluation tool in clinical medicine. Clin Chem 1993;39(4):561-577.

[12] Bollmann A, et al. Analysis of surface electrocardiograms in atrial fibrillation: techniques, research, and clinical applications. EP Europace 2006;8(11):911-926.

[13] Lankveld T, et al. The ECG as a tool to determine atrial fibrillation complexity. Heart 2014;100(14):1077-1084.

[14] Park J, et al. Early differentiation of long-standing persistent atrial fibrillation using the characteristics of fibrillatory waves in surface ECG multi-leads. Sci Rep 2019;9(1):2746.

[15] Zeemering $S$, et al. The electrocardiogram as a predictor of successful pharmacological cardioversion and progression of atrial fibrillation. Europace 2018;20(7):e96-e104.

[16] Lankveld T, et al. Atrial fibrillation complexity parameters derived from surface ECGs predict procedural outcome and long-term follow-up of stepwise catheter ablation for atrial fibrillation. Circ Arrhythm Electrophysiol 2016;9(2).

Address for correspondence:

Raúl Alcaraz Martínez

Technical School of Cuenca, Campus Universitario s/n, 16071, Cuenca, Spain

Phone: +34-969-179-100 Ext. 4847

e-mail: raul.alcaraz@uclm.es 